\title{
Fite-Wintner-Leighton-Type Oscillation Criteria for Second-Order Differential Equations with Nonlinear Damping
}

\author{
Mervan Pašić \\ Department of Mathematics, Faculty of Electrical Engineering and Computing, University of Zagreb, 10000 Zagreb, Croatia \\ Correspondence should be addressed to Mervan Pašić; mervan.pasic@gmail.com
}

Received 15 October 2012; Accepted 29 December 2012

Academic Editor: Yuriy Rogovchenko

Copyright (C) 2013 Mervan Pašić. This is an open access article distributed under the Creative Commons Attribution License, which permits unrestricted use, distribution, and reproduction in any medium, provided the original work is properly cited.

\begin{abstract}
Some new oscillation criteria for a general class of second-order differential equations with nonlinear damping are shown. Except some general structural assumptions on the coefficients and nonlinear terms, we additionally assume only one sufficient condition (of Fite-Wintner-Leighton type). It is different compared to many early published papers which use rather complex sufficient conditions. Our method contains three items: classic Riccati transformations, a pointwise comparison principle, and a blow-up principle for sub- and supersolutions of a class of the generalized Riccati differential equations associated to any nonoscillatory solution of the main equation.
\end{abstract}

\section{Introduction}

In the paper, we develop some new oscillation criteria for the following class of second-order differential equations with nonlinear damping:

$$
\begin{aligned}
& \left(r(t) k_{1}\left(x, x^{\prime}\right)\right)^{\prime}+p(t) k_{2}\left(x, x^{\prime}\right) x^{\prime} \\
& \quad+q(t) f(x)=0, \quad t \geq t_{0}>0,
\end{aligned}
$$

where the coefficients $r \in C^{1}\left(\left[t_{0}, \infty\right),(0, \infty)\right), p, q \in C\left(\left[t_{0}\right.\right.$, $\infty), \mathbb{R})$, and the functions $k_{1}(u, v), k_{2}(u, v)$ are continuous in all their variables, $k_{1} \in C^{1}\left(\mathbb{R}^{2}, \mathbb{R}\right)$ and solution $x=x(t), x \in$ $C^{2}\left(\left(t_{0}, \infty\right), \mathbb{R}\right) \cap C\left(\left[t_{0}, \infty\right), \mathbb{R}\right)$. A function $x(t)$ is said to be oscillatory if there is a sequence $t_{n} \geq t_{0}$ such that $x\left(t_{n}\right)=0$ and $t_{n} \rightarrow \infty$ as $n \rightarrow \infty$.

In Section 2, we present some basic structural assumptions on the coefficients: $r(t), p(t)$, and $q(t)$ and on the nonlinear functions: $k_{1}(u, v)$ and $k_{2}(u, v)$, which are slightly more general than those of the previously published results such as in Zhao et al. [1, Theorem 2.1] (see also Theorem A, Section 2), [1, Theorems 2.2-2.8], [2, Theorem 2], [3, Theorem 2.1]. In Section 3, we study some new oscillation criteria for (1) based on an additional sufficient condition of Fite-Wintner-Leighton type, which is rather simpler than Kamenev-type conditions or related complex ones. Equation
(1) in various different forms has been considered in many several published papers, see, for instance, [4-12] and references therein. In Section 4, we state and prove a pointwise comparison principle between all sub- and supersolutions of the corresponding generalized Riccati differential equation associated with every nonoscillatory solution $x(t)$ of (1). Furthermore, under the main assumption of Fite-WintnerLeighton type, we construct a subsolution of the Riccati differential equation which blows up in time. It together with classic Riccati transformation gives the proof of the main result.

\section{Main Assumptions and Remarks}

In particular, for $m=n=1$, in [1] authors firstly supposed the next five basic conditions on the coefficients $p(t), q(t)$ and the functions $f(u), k_{1}(u, v)$, and $k_{2}(u, v)$ :

$$
p(t) \geq 0 \quad \forall t \geq t_{0},
$$

$$
\begin{array}{r}
\frac{f(u)}{u} \geq K \quad \text { for some } K>0 \text { and all } u \in \mathbb{R}, u \neq 0, \\
q(t) \geq 0 \quad \forall t \geq t_{0}, \\
q(t) \neq 0 \text { on }\left[t_{*}, \infty\right) \text { for any } t_{*} \geq t_{0},
\end{array}
$$




$$
\begin{array}{r}
k_{1}^{2 m}(u, v) \leq \alpha_{1} u^{2 m-2} v k_{1}(u, v) \quad \text { for some } m \in \mathbb{N}, \alpha_{1}>0 \\
\text { and all }(u, v) \in \mathbb{R}^{2}, u \neq 0, \\
u^{2 n-1} v k_{2}(u, v) \geq \alpha_{2} k_{1}^{2 n}(u, v) \quad \text { for some } n \in \mathbb{N}, \alpha_{2}>0 \\
\text { and all }(u, v) \in \mathbb{R}^{2}, u \neq 0 .
\end{array}
$$

Such a set of assumptions, with slightly different (6) and $m=$ $n=1$, was introduced for the first time in [2], see also [3]. Just the same as in [1], besides (6) we also consider a similar assumption:

$$
u v k_{2}(u, v) \geq \alpha_{2} u k_{1}(u, v) \quad \text { for some } \alpha_{2}>0 \text { and all }(u, v) \in \mathbb{R}^{2} \text {. }
$$

And, in the case when $p(t)$ and $q(t)$ may change the sign, instead of (2)-(4) and (6), one considers also:

$$
\begin{aligned}
& f \in C^{1}(\mathbb{R}, \mathbb{R}), \quad \text { uf }(u) \neq 0, \\
& f^{\prime}(u) \geq K>0 \quad \forall u \in \mathbb{R}, u \neq 0,
\end{aligned}
$$

$v k_{2}(u, v)=\alpha_{2} k_{1}(u, v)$ for some $\alpha_{2}>0$ and all $(u, v) \in \mathbb{R}^{2}$.

Here, assumptions (5) and (6) are generalized in the following sense, see Theorem 5-(ii) and (iii), respectively,

$$
\begin{gathered}
v k_{1}(u, v) \geq 0 \quad \forall(u, v) \in \mathbb{R}^{2}, \\
u v k_{2}(u, v) \geq 0 \quad \forall(u, v) \in \mathbb{R}^{2},
\end{gathered}
$$

which are weaker than (5) and (6), respectively. One of the reasons for that is presented in the next remarks.

\section{Remark 1}

(1) The most simple second-order differential operator which satisfies assumption (5) for $m=1$ is linear in variable $v$; that is,

$$
\left(r(t) k_{1}\left(x, x^{\prime}\right)\right)^{\prime}=\left(A(x) x^{\prime}\right)^{\prime}
$$

where $r(t) \equiv 1, k_{1}(u, v)=A(u) v$, and $A(u)$ is an arbitrary function satisfying $0 \leq A(u) \leq \alpha_{1}$. It is because $k_{1}^{2}(u, v)=A^{2}(u) v^{2} \leq \alpha_{1} A(u) v^{2}=\alpha_{1} v k_{1}(u, v)$ for all $(u, v) \in \mathbb{R}^{2}$ and $\alpha_{1} \geq 0$, see also Corollary 6 . However, it is easy to check that the differential operator from (7) does not satisfy assumption (5) for every $m>1$.

(2) Next, we consider the corresponding second-order quasilinear differential operator:

$$
\left(r(t) k_{1}\left(x, x^{\prime}\right)\right)^{\prime}=\left(A(x)\left|x^{\prime}\right|^{\beta-1} x^{\prime}\right)^{\prime}
$$

where $r(t) \equiv 1, k_{1}(u, v):=A(u)|v|^{\beta-1} v$, and $A(u)$ is an arbitrary function satisfying $0 \leq A(u) \leq \alpha_{1}$ and in order to ensure that $k_{1} \in C^{1}\left(\mathbb{R}^{2}, \mathbb{R}\right)$, we take $\beta \geq 1$ since $\partial k_{1} / \partial v=\beta|v|^{\beta-1}$. Unfortunately, the differential operator from (8) does not satisfy assumption (5) for every $m \in \mathbb{N}, \beta>1$. It is because $k_{1}^{2}(u, v)=A^{2}(u) v^{2 \beta-2} v^{2}=A^{2}(u) v^{2 \beta} \leq \alpha_{1} A(u) v^{2 \beta}=$ $\alpha_{1} v k_{1}(u, v)|v|^{\beta-1}$, which is different from (5).

(3) Unlike (5), the differential operator from (8) satisfies assumption (5) $)_{w}$, and hence, (8) is also included in our study of the oscillation of (1), see Corollary 11.

(4) Although both differential operators from (7) and (8) do not satisfy assumption (5) for every $m>1$, the so-called generalized prescribed mean curvature-like differential operator:

$$
\left(r(t) k_{1}\left(x, x^{\prime}\right)\right)^{\prime}=\left(A(x) \frac{x^{\prime}}{\left(1+x^{\prime 2}\right)^{\alpha / 2}}\right)^{\prime}
$$

satisfies assumption (5) for every $m \geq 1$, where $r(t) \equiv$ $1, k_{1}(u, v):=A(u) v /\left(1+v^{2}\right)^{\alpha / 2}, \alpha \geq 1$, and $A(u)$ is an arbitrary function satisfying $0 \leq A^{2 m-1}(u) \leq \alpha_{1} u^{2 m-2}$, see Corollary 9.

(5) The simple case $k_{2}(u, v) \equiv 0$ is involved in $(6)_{w}$ unlike (6), and hence, the nonlinear equation $x^{\prime \prime}+q(t) f(x)=$ 0 can be considered as a special case of (1).

We pay attention to the recently published paper [13] in which authors show that any generalization of the assumptions (2)-(6) should be done very carefully.

Now, we can recall [1, Theorems 2.5].

Theorem A. Let (2)-(6) hold. Assume that there exist $\rho \in$ $C^{1}\left(\left[t_{0}, \infty\right),(0, \infty)\right), H \in \mathbb{H}, g \in C^{1}\left(\left[t_{0}, \infty\right), \mathbb{R}\right)$, and some $t_{1} \geq t_{0}$ such that for all $T \geq t_{1}$ :

$$
\begin{aligned}
\limsup _{t \rightarrow \infty} \frac{1}{H(t, T)} \int_{T}^{t}\left[H(t, s) \gamma_{1}(s)\right. \\
\left.\quad-\frac{\alpha_{1} \rho(s) v(s) r^{2}(s)}{4\left(\alpha_{2} p(s)+r(s)\right)} Q_{1}^{2}(t, s)\right] d s=\infty
\end{aligned}
$$

where $v(t)$ and $\gamma_{1}(t)$ are defined, respectively, by

$$
\begin{aligned}
& v(t)= \exp \left(-\frac{2}{\alpha_{1}} \int_{t_{1}}^{t} g(s) d s\right), \\
& \gamma_{1}(t)=\rho(t) v(t)\left[\frac{1}{\alpha_{1}} r(t) g^{2}(t)\right. \\
&\left.+K q(t)+\alpha_{2} g^{2}(t) p(t)-(r(t) g(t))^{\prime}\right],
\end{aligned}
$$


and $Q_{1} \in C(D, \mathbb{R})$ satisfies

$$
\begin{aligned}
-\frac{\partial H(t, s)}{\partial s}= & \left(\frac{\rho^{\prime}(s)}{\rho(s)}+\frac{2 \alpha_{2} p(s) g(s)}{r(s)}\right) H(t, s) \\
& +Q_{1}(t, s) \sqrt{H(t, s)} .
\end{aligned}
$$

Then, (1) is oscillatory.

In Theorem $\mathrm{A}$, the set $D=\left\{(t, s): t \geq s \geq t_{0}\right\}$. And the assumption $H \in \mathbb{M}$ means that $H \in C\left(D, \mathbb{R}_{+}\right)$, $\partial H(t, s) / \partial s$ is continuous on $D_{0}=\left\{(t, s): t>s \geq t_{0}\right\}$, $H(t, t)=0$ for all $t \geq t_{0}$ and $H(t, s)>0$ for all $(t, s) \in$ $D_{0}$. It is easy to see that the coefficients: $r(t), p(t)$, and $q(t)$ are involved in the assumptions (10)-(12), often called the general Kamenev-type conditions, about the Kamenevtype conditions and their several generalization we refer the reader, for instance, to [14-18]. The main purpose of supposing the existence of the functions: $\rho(t), H(t, s)$, and $g(t)$ satisfying the corresponding assumptions (10)-(12) is to ensure the nonexistence of continuous function $w(t)$ which satisfies the corresponding Riccati differential inequality:

$$
w^{\prime} \geq \alpha(t) \omega^{2}+\beta(t) \omega+\gamma(t), \quad t \geq T,
$$

where $\alpha(t), \beta(t)$, and $\gamma(t)$ depend on $r(t), p(t), \alpha_{1}$, and $\alpha_{2}$, and $T \geq t_{0}$.

Instead of Kamenev-type conditions (10)-(12), we consider the next one (which can be called the Fite-WintnerLeighton-type condition by a reason given in Remark 2): for the explicitly given two functions $a(t)$ and $b(t)$ which depend on the data $r(t), p(t), m, n, \alpha_{1}, \alpha_{2}$, and $q(t)$, (see Theorems 5 and 15), let there be a function $E(t)$ and a point $T_{1} \geq t_{0}$ such that

$$
\begin{gathered}
E \in C\left(\left[T_{1}, \infty\right)\right), \quad \lim _{t \rightarrow \infty} \sup _{T_{1}}^{t} E(\tau) d \tau=\infty, \\
\forall m, n \in \mathbb{N}, \text { we have } \\
E(t) \leq \begin{cases}\min \{a(t), b(t)\}, t \geq T_{1}, & \text { if } \min \{m, n\}=1, \\
\min \{a(t), b(t)-a(t)\}, t \geq T_{1}, & \text { if } \min \{m, n\}>1 .\end{cases}
\end{gathered}
$$

Combining a pointwise comparison principle and a blowup argument, which is a different method than that in the case of Kamenev-type conditions, we are able to prove the nonexistence of any continuous function $\psi(t)$ which satisfies the corresponding Riccati differential inequality:

$$
\psi^{\prime} \geq a_{1}(t) \psi^{2 m}+a_{2}(t) \psi^{2 n}+b(t), \quad t \geq T,
$$

where $a_{1}(t), a_{2}(t)$, and $b(t)$ are arbitrary functions. On the various aspects of the comparison principles, we refer the reader, for instance, to $[19,20]$-the comparison principles for Volterra integral operators, $[21,22]$ - the pointwise comparison principle for ODEs and [23] - the abstract form of comparison principles.

Remark 2. It is simple to check that in particular for $k_{1}(u, v) \equiv$ $v, k_{2}(u, v) \equiv 0$, and $f(u) \equiv u$, the conditions (3), (5) with $m=1$ and $(6)_{w}$ still hold where the inequality " $\geq$ " is replaced by "=." Then (1) becomes the linear second-order differential equation (LEq): $\left(r(t) x^{\prime}\right)^{\prime}+q(t) x=0$. Hence, the inequality in (14) for $m=n=1$ can be replaced by the corresponding equality, where $a(t)=1 / r(t)$ and $b(t)=q(t)$ (see the case (iii) of Theorem 5), and so, we conclude that in this case, (14) is equivalent to:

$$
\lim \sup _{t \rightarrow \infty} \int_{T_{1}}^{t} \frac{1}{r(\tau)} d \tau=\lim \sup _{t \rightarrow \infty} \int_{T_{1}}^{t} q(\tau) d \tau=\infty,
$$

which presents the classic Fite-Wintner-Leighton oscillation criterion for linear second-order differential equation (LEq), where "lim" appears instead of "lim sup." In Fite [24], Wintner [25], and Leighton [26] equation (LEq) was considered, respectively, with $r(t) \equiv 1$ and $q(t)>0, r(t) \equiv 1$ and $q(t)$ may change sign, and arbitrary $r(t)>0$ and $q(t)$ may change sign. Nonlinear version of such a class of oscillation criteria was due to Wong [27], and Nth-order extension for linear equations can be found in Travis [28].

In order to simplify notation, we firstly introduce the following definition for the pointwise comparison principle of the corresponding Riccati differential equation:

$$
w^{\prime}=a_{1}(t) w^{2 m}+a_{2}(t) w^{2 n}+b(t), \quad t \geq T
$$

where $a_{1}(t), a_{2}(t)$, and $b(t)$ are three arbitrary functions, and $T \geq t_{0}$.

Definition 3. Let $T_{0}$ and $T^{*}$ be two arbitrary real numbers, $T \leq T_{0}<T^{*}$. Two functions, $\varphi(t)$ and $\psi(t), \varphi, \psi \in$ $C^{1}\left(\left(T_{0}, T^{*}\right), \mathbb{R}\right) \cap C\left(\left[T_{0}, T^{*}\right), \mathbb{R}\right)$, are said to be, respectively, subsolution and supersolution of the Riccati differential equation (17) provided that

$$
\begin{gathered}
\varphi^{\prime} \leq a_{1}(t) \varphi^{2 m}+a_{2}(t) \varphi^{2 n}+b(t), \\
\psi^{\prime} \geq a_{1}(t) \psi^{2 m}+a_{2}(t) \psi^{2 n}+b(t), \quad t \in\left(T_{0}, T^{*}\right) .
\end{gathered}
$$

Moreover, if the statement:

$$
\varphi\left(T_{0}\right) \leq \psi\left(T_{0}\right) \text { implying } \varphi(t) \leq \psi(t) \quad \forall t \in\left[T_{0}, T^{*}\right)
$$

is fulfilled for all sub- and supersolutions $\varphi, \psi \in C^{1}\left(\left(T_{0}\right.\right.$, $\left.\left.T^{*}\right), \mathbb{R}\right) \cap C\left(\left[T_{0}, T^{*}\right), \mathbb{R}\right)$ of $(17)$, then we say that comparison principle (19) holds for (17) with arbitrary $T_{0}$ and $T^{*}, T \leq$ $T_{0}<T^{*}$.

Remark 4. The possibilty that (19) holds for all sub- and supersolutions and with arbitrary $T_{0}$ and $T^{*}, T \leq T_{0}<T^{*}$ plays an essential role in some concrete situations. According to it, when the comparison principle (19) holds for the Riccati differential equation (17) with arbitrary $T_{0}$ and $T^{*}$, $T \leq T_{0}<T^{*}$, then we can choose some concrete suband supersolutions as well as $T_{0}$ and $T^{*}$ with some suitable properties. 
Our method contains the next three steps:

(i) at the first step, we give a sufficient condition on $a_{1}(t), a_{2}(t)$ such that comparison principle (19) holds for the Riccati differential equation (17) with arbitrary $b(t), T_{0}$ and $T^{*}, T \leq T_{0}<T^{*} ;$

(ii) at the second step, for a supersolution $\psi \in C^{1}((T$, $\infty), \mathbb{R}) \cap C([T, \infty), \mathbb{R})$ of $(17)$, where $a_{1}(t), a_{2}(t)$, and $b(t)$ are three arbitrary functions, and under assumption (14), we find two real numbers $T_{0}$ and $T^{*}$, $T \leq T_{0}<T^{*}$, and construct a subsolution $\varphi(t), \varphi \in$ $C^{1}\left(\left(T_{0}, T^{*}\right), \mathbb{R}\right) \cap C\left(\left[T_{0}, T^{*}\right), \mathbb{R}\right)$ of (17) such that the following initial and blow-up arguments are satisfied:

$$
\varphi\left(T_{0}\right) \leq \psi\left(T_{0}\right), \quad \lim _{t \rightarrow T^{*}} \varphi(t)=\infty ;
$$

(iii) at the third step, under conditions (2)-(6) or related ones such as $(5)_{w}$ and $(6)_{w}$, we show that if the main equation (1) allows a nonoscillatory solution $x(t)$, then the function:

$$
\psi(t)=-\frac{r(t) k_{1}\left(x(t), x^{\prime}(t)\right)}{x(t)}, \quad t \geq T,
$$

is well defined for some $T \geq t_{0}, \psi \in C^{1}((T, \infty), \mathbb{R}) \cap$ $C([T, \infty), \mathbb{R})$, and $\psi(t)$ is a supersolution of (17) with some concrete $a_{1}(t), a_{2}(t)$, and $b(t)$; in the case when $p(t)$ and $q(t)$ change the sign, instead of (21), we consider the function:

$$
\psi(t)=-\frac{r(t) k_{1}\left(x(t), x^{\prime}(t)\right)}{f(x(t))}, \quad t \geq T .
$$

In conclusion, combining (19) and (20), we obtain the nonexistence of any continuous supersolution of the Riccati differential equation (17), and hence, the function $\psi(t)$ given by (21) or (22) is not possible. Therefore, (1) does not allow any nonoscillatory solution.

\section{Main Results and Examples}

As usual, we recognize two main different cases: the first one is when $p(t)$ and $q(t)$ are positive and the second one is when they may change the sign. Moreover, in the first case, depending on the combination of assumptions (5), (6), (5) $(6)_{w}$, and $(6)_{1}$, we consider five subcases such as is done in our first oscillation criterion for (1).

Theorem 5 (positive coefficients). Let assumptions (2)-(4) be fulfilled. Then, (1) is oscillatory if one of the next five cases is met.

(i) Let $m, n \in \mathbb{N}$ and (5), (6) hold. One supposes (14) with respect to $a(t):=a_{1}(t)+a_{2}(t)$ provided that $m=n=1$ or $a(t):=\min \left\{a_{1}(t), a_{2}(t)\right\}$, otherwise,

$$
a_{1}(t)=\frac{1}{\alpha_{1} r^{2 m-1}(t)}, \quad a_{2}(t)=\frac{p(t) \alpha_{2}}{r^{2 n}(t)}, \quad b(t)=K q(t) \text {. }
$$

(ii) Let $n \in \mathbb{N}$ and (5) $)_{w}$, (6) hold. One supposes (14) with respect to $a(t)$ and $b(t)$ given by

$$
a(t)=\frac{p(t) \alpha_{2}}{r^{2 n}(t)}, \quad b(t)=K q(t) .
$$

(iii) Let $m \in \mathbb{N}$ and (5), (6) w hold. One supposes (14) with respect to $a(t)$ and $b(t)$ given by

$$
a(t)=\frac{1}{\alpha_{1} r^{2 m-1}(t)}, \quad b(t)=K q(t) .
$$

(iv) Let $m=1$ and (5), (6) 1 hold. One supposes (14) with respect to $a(t)$ and $b(t)$ given by

$$
\begin{aligned}
& a(t)=\frac{1}{\alpha_{1} r(t)} e^{-\alpha_{2} \int(p(\tau) / r(\tau)) d \tau}, \\
& b(t)=K q(t) e^{\alpha_{2} \int(p(\tau) / r(\tau)) d \tau} .
\end{aligned}
$$

(v) Let $p \in C^{1}\left(\left(t_{0}, \infty\right), \mathbb{R}\right), m=1$, and (5), (6) ${ }_{1}$ hold. One supposes (14) with respect to $a(t)$ and $b(t)$ given by

$$
a(t)=\frac{1}{\alpha_{1} r(t)}, \quad b(t)=K q(t)-\frac{\alpha_{1} \alpha_{2} p^{\prime}(t)}{2}-\frac{\alpha_{1} \alpha_{2}^{2} p^{2}(t)}{4 r(t)} .
$$

For each of the cases (i)-(v) of Theorem 5, we derive some consequences and examples, which show the importance of our oscillation criterion.

The case (i) of Theorem 5 for $m=n=1$ allows us to consider the following class of equations:

$$
\left(r(t) A(x) x^{\prime}\right)^{\prime}+p(t) B(x) x^{\prime 2}+q(t) f(x)=0, \quad t \geq t_{0}>0,
$$

where the functions $A=A(u), A \in C^{1}(\mathbb{R})$, and $B(u)$ satisfy

$$
0 \leq A(u) \leq \alpha_{1}, \quad u B(u) \geq \alpha_{2} A^{2}(u) \quad \forall(u, v) \in \mathbb{R}^{2},
$$

for some $\alpha_{1}>0$ and $\alpha_{2}>0$. Under assumption (29), it is easy to see that the functions $k_{1}(u, v):=A(u) v$ and $k_{2}(u, v):=$ $B(u) v$ satisfy both required assumptions (5) and (6) with $m=$ $n=1$. Hence, as an easy consequence of Theorem 5 , we obtain the following result.

Corollary 6. Let (2)-(4) and (14) hold with respect to $a(t)$ and $b(t)$ given in case ( $i)$ of Theorem 5 with $m=n=1$. If $A(u)$ and $B(u)$ satisfy (29), then (28) is oscillatory.

Example 7. Let $K>0, \mu \leq 1$ or $v \geq 2 \mu-1$, and $\sigma \leq 1$. Then, the equation:

$$
\left(t^{\mu} \frac{x^{2}}{1+x^{2}} x^{\prime}\right)^{\prime}+t^{v} x^{3} x^{\prime 2}+K t^{-\sigma} x=0, \quad t \geq t_{0}>0
$$

is oscillatory. Indeed, it is enough to check that the coefficients $r(t)=t^{\mu}, p(t)=t^{v}$, and $q(t)=t^{-\sigma}$ and the functions $f(u)=K u, A(u)=u^{2} /\left(1+u^{2}\right)$, and $B(u)=u^{3}$ satisfy all the assumptions of Corollary 6 with respect to $\alpha_{1}=\alpha_{2}=1$ and $E(t)=c / t$ for some $c>0$. 
Example 8. Let $K>0, \mu \leq 1$ or $v \geq 2 \mu-1$, and $\sigma \leq 1$. Then, the equation:

$$
\left(t^{\mu}(\sin x)^{2} x^{\prime}\right)^{\prime}+t^{v} x^{3} x^{\prime 2}+K t^{-\sigma} x=0, \quad t \geq t_{0}>0
$$

is oscillatory. In fact, it is easy to check that the coefficients $r(t)=t^{\mu}, p(t)=t^{v}$, and $q(t)=t^{-\sigma}$ and the functions $f(u)=$ $K u, A(u)=\sin ^{2} u$, and $B(u)=u^{3}$ satisfy all the assumptions of Corollary 6 with respect to $\alpha_{1}=\alpha_{2}=1$ and $E(t)=c / t$ for some $c>0$.

The case (i) of Theorem 5 for $m, n \in \mathbb{N}$ proposes the following class of differential equations:

$$
\begin{aligned}
& \left(r(t) A(x) \frac{x^{\prime}}{\left(1+x^{\prime 2}\right)^{\alpha / 2}}\right)^{\prime}+p(t) B(x)\left(\frac{x^{\prime}}{\left(1+x^{\prime 2}\right)^{\alpha / 2}}\right)^{2 n} \\
& \quad+q(t) f(x)=0, \quad t \geq t_{0}>0,
\end{aligned}
$$

where $\alpha \geq 1, n \in \mathbb{N}$, and the functions $A=A(u), A \in C^{1}(\mathbb{R})$, and $B(u)$ satisfy

$$
0 \leq A^{2 m-1}(u) \leq \alpha_{1} u^{2 m-2}, \quad u^{2 n-1} B(u) \geq \alpha_{2} A^{2 n}(u), \quad u \in \mathbb{R} .
$$

As a consequence of Theorem 5, we derive the next interesting corollary.

Corollary 9. Let (2)-(4) and (14) hold with respect to $a(t)$ and $b(t)$ given in case ( $i)$ of Theorem 5. If $A(u)$ and $B(u)$ satisfy (33), then (32) is oscillatory.

Example 10. Let $\alpha \geq 1, n \in \mathbb{N}, K>0, \mu \leq 1$ or $v \geq 2 \mu-1$, and $\sigma \leq 1$. Then, according to Corollary 9 , we conclude that the equation:

$$
\begin{aligned}
& \left(t^{\mu} \frac{x^{2}}{1+x^{2}} \frac{x^{\prime}}{\left(1+x^{\prime 2}\right)^{\alpha / 2}}\right)^{\prime}+t^{v} x\left(\frac{x x^{\prime}}{\left(1+x^{2}\right)\left(1+x^{\prime 2}\right)^{\alpha / 2}}\right)^{2 n} \\
& +K t^{-\sigma} x=0, \quad t \geq t_{0}>0
\end{aligned}
$$

is oscillatory.

We have pointed out in Remark 1 that assumption $(5)_{w}$ unlike (5) allows to consider the oscillation of the following quasilinear differential equation:

$$
\begin{aligned}
& \left(r(t) A(x)\left|x^{\prime}\right|^{\beta-1} x^{\prime}\right)^{\prime}+p(t) B(x) x^{\prime 2 \beta} \\
& \quad+q(t) f(x)=0, \quad t \geq t_{0}>0,
\end{aligned}
$$

where $\beta \geq 1$ and the functions $A=A(u), A \in C^{1}(\mathbb{R})$, and $B(u)$ satisfy

$$
0 \leq A(u), \quad u B(u) \geq \alpha_{2} A^{2}(u) \quad \forall(u, v) \in \mathbb{R}^{2},
$$

for some $\alpha_{2}>0$. It is clear that (28) is a particular case of (35) for $\beta=1$. Under assumption (36), the functions

$$
k_{1}(u, v):=A(u)|v|^{\beta-1} v, \quad k_{2}(u, v):=B(u) v^{2 \beta-1}
$$

satisfy both required assumptions (5) $w$ and (6) with $n=1$. Therefore, we can derive the following easy consequence of the case (ii) of Theorem 5 .

Corollary 11. Let (2)-(4) and (14) hold with respect to $a(t)$ and $b(t)$ given in case (ii) of Theorem 5 with $n=1$. If $A(u)$ and $B(u)$ satisfy (36), then (35) is oscillatory.

Example 12. Let $\beta \geq 1, K>0, v \geq 2 \mu-1$ and $\sigma \leq 1$. Then the equation:

$$
\left(t^{\mu}(\sin x)^{2} x^{\prime \beta}\right)^{\prime}+t^{v} x^{3} x^{\prime 2 \beta}+K t^{-\sigma} x=0, \quad t \geq t_{0}>0,
$$

is oscillatory. In fact, it is enough to check that the coefficients $r(t)=t^{\mu}, p(t)=t^{v}, q(t)=t^{-\sigma}$ and the functions $f(u)=$ $K u, A(u)=\sin ^{2} u$ and $B(u)=u^{3}$ satisfy all assumptions of Corollary 11 with respect to $E(t)=c / t$ for some $c>0$.

The case (iii) of Theorem 5 allows us to consider the following class of equations:

$$
\begin{gathered}
\left(r(t) A(x) x^{\prime}\right)^{\prime}+p(t) B(x) C\left(x^{\prime}\right) x^{\prime} \\
\quad+q(t) f(x)=0, \quad t \geq t_{0}>0
\end{gathered}
$$

where the functions $A=A(u), A \in C^{1}(\mathbb{R}), B(u)$ and $C(v)$ satisfy:

$$
0 \leq A(u) \leq \alpha_{1}, \quad u B(u) \geq 0, \quad v C(v) \geq 0 \quad \forall(u, v) \in \mathbb{R}^{2},
$$

for some $\alpha_{1}>0$. Under this assumption, it is easy to see that the functions $k_{1}(u, v):=A(u) v$ and $k_{2}(u, v):=B(u) C(v)$ satisfy both required assumptions (5) and (6) $)_{w}$. Hence, as an easy consequence of case (iii) of Theorem 5 , we obtain the following result.

Corollary 13. Let (2), (3), and (14) hold with respect to a(t) given in case (iii) of Theorem 5. If $A(u), B(u)$, and $C(v)$ satisfy (40), then (39) is oscillatory.

Example 14. Let $K>0, \mu \leq 1, \nu \geq 0, \lambda \geq 0$, and $\sigma \leq 1$. Then, the equation:

$$
\left(t^{\mu} \frac{x^{2}}{1+x^{2}} x^{\prime}\right)^{\prime}+t^{\nu}|x|^{\lambda} x \operatorname{sh}\left(x^{\prime}\right) x^{\prime}+K t^{-\sigma} x=0, \quad t \geq t_{0}>0
$$

is oscillatory. In order to show that, it is enough to check that the coefficients: $r(t)=t^{\mu}, q(t)=t^{-\sigma}$ and the functions: $f(u)=$ $K u, A(u)=u^{2} /\left(1+u^{2}\right), B(u)=|u|^{\lambda} u$, and $C(v)=\operatorname{sh}(v)$ satisfy all the assumptions of Corollary 13 with respect to $\alpha_{1}=$ $\alpha_{2}=1$ and $E(t)=c / t$ for some $c>0$. 
Next, we consider the oscillation of (1) in the case when the coefficients $p(t)$ and $q(t)$ may change the sign.

Theorem 15 (coefficients may change the sign). Let $m=1$ and assumptions $(3)_{1},(5)$, and $(6)_{2}$ hold. Then, (1) is oscillatory provided that one of the following two cases is met. (vi) One assumes (14) with respect to $a(t)$ and $b(t)$ given by

$$
\begin{aligned}
& a(t)=\frac{K}{\alpha_{1} r(t)} e^{-\alpha_{2} \int_{T}^{t}(p(\tau) / r(\tau)) d \tau}, \\
& b(t)=q(t) e^{\alpha_{2} \int_{T}^{t}(p(\tau) / r(\tau)) d \tau}, \quad t \geq T .
\end{aligned}
$$

(vii) Let $p \in C^{1}\left(\left(t_{0}, \infty\right), \mathbb{R}\right)$. One assumes (14) with respect to $a(t)$ and $b(t)$ given by

$$
a(t)=\frac{K}{\alpha_{1} r(t)}, \quad b(t)=q(t)-\frac{\alpha_{1} \alpha_{2} p^{\prime}(t)}{2 K}-\frac{\alpha_{1} \alpha_{2}^{2} p^{2}(t)}{4 K r(t)}, \quad t \geq T .
$$

The case (vi) of Theorem 15 allows us to consider the following class of equations:

$$
\left(r(t) A(x) x^{\prime}\right)^{\prime}+\alpha_{2} p(t) A(x) x^{\prime}+q(t) f(x)=0, \quad t \geq t_{0}>0,
$$

where $\alpha_{2} \in \mathbb{R}$, and the function $A(u)$ satisfies

$$
0 \leq A(u) \leq \alpha_{1} \quad \forall(u, v) \in \mathbb{R}^{2} \text { and some } \alpha_{1}>0 .
$$

Under (45), one can easily check that the functions $k_{1}(u, v):=$ $A(u) v$ and $k_{2}(u, v):=\alpha_{2} A(u)$ satisfy both required assumptions (5) and $(6)_{2}$. Hence, as an easy consequence of case (vi) of Theorem 5, we conclude the next consequence.

Corollary 16. Let $(3)_{1}$ and (14) hold with respect to $a(t)$ and $b(t)$ given in case ( $v i)$ of Theorem 5 . If $A(u)$ satisfies (45), then (44) is oscillatory.

Example 17. Let $\mu \geq 2$ and $q_{0} \in \mathbb{R}$. Then, the equations:

$$
\begin{gathered}
\left(t^{-\mu} \frac{x^{2}}{1+x^{2}} x^{\prime}\right)^{\prime}+t^{-\mu-1} \frac{x^{2}}{1+x^{2}} x^{\prime} \\
+q_{0}(\sin t) x=0, \quad t \geq t_{0}>0, \\
\left(t^{-\mu}(\sin x)^{2} x^{\prime}\right)^{\prime}+t^{-\mu-1}(\sin x)^{2} x^{\prime} \\
+q_{0}(\sin t) x=0, \quad t \geq t_{0}>0
\end{gathered}
$$

are oscillatory. In order to show that, it is enough to check that the coefficients: $r(t)=t^{-\mu}, p(t)=t^{-\mu-1}$, and $q(t)=q_{0} \sin t$ and the functions: $f(u)=u, A(u)=u^{2} /\left(1+u^{2}\right)$, and $A(u)=$ $\sin ^{2} u$ satisfy all the assumptions of Corollary 16 with respect to $\alpha_{1}=\alpha_{2}=1$ and $E(t)=c t \sin t$ for some $c \in \mathbb{R}, c \neq 0$.

\section{Proofs of the Main Results}

In this section, we study the oscillation of (1) in the view of a pointwise comparison principle presented below, which will be shown for the corresponding Riccati differential equation.
Definition 18. A function $h(t, u)$ is said to be locally Lipschitz in the second variable if for any bounded interval $I_{0} \subseteq[T, \infty)$ and $M>0$ there is a constant $L>0$ depending on $I_{0}, M, h$ such that

$$
\begin{gathered}
\left|h\left(t, u_{1}\right)-h\left(t, u_{2}\right)\right| \leq L\left|u_{1}-u_{2}\right| \quad \forall t \in I_{0}, \\
u_{1}, u_{2} \in[-M, M] .
\end{gathered}
$$

Now, we state and use the following general comparison principle, which will be proved at the end of this section.

Lemma 19. Let $T_{0}$ and $T^{*}$ be two arbitrary real numbers such that $T_{0}<T^{*}$. Let $\tilde{\varphi}(t)$ and $\tilde{\psi}(t), \tilde{\varphi}, \tilde{\psi} \in C^{1}\left(\left(T_{0}, T^{*}\right), \mathbb{R}\right) \cap$ $C\left(\left[T_{0}, T^{*}\right), \mathbb{R}\right)$, be two functions satisfying:

$$
\tilde{\varphi}^{\prime} \leq h(t, \widetilde{\varphi}), \quad \widetilde{\psi}^{\prime} \geq h(t, \widetilde{\psi}), \quad t \in\left(T_{0}, T^{*}\right),
$$

where $h(t, u)$ is a locally Lipschitz function in the second variable. Then, we have

$$
\widetilde{\varphi}\left(T_{0}\right) \leq \widetilde{\psi}\left(T_{0}\right) \text { implies } \widetilde{\varphi}(t) \leq \widetilde{\psi}(t) \quad \forall t \in\left[T_{0}, T^{*}\right) .
$$

Definition 20. A function $a(t)$ is said to be locally bounded on $[T, \infty)$, if for any bounded interval $I_{0} \subseteq[T, \infty)$ there is a constant $C>0$ depending on $I_{0}$ such that $|a(t)| \leq C$ for all $t \in I_{0}$.

According to Lemma 19, we are able to give a sufficient condition on the functions: $a_{1}(t), a_{2}(t)$ such that the Riccati differential equation (17) satisfies the comparison principle (19).

Lemma 21. If $a_{1}(t)$ and $a_{2}(t)$ are two locally bounded functions on $[T, \infty)$, then comparison principle (19) holds for the Riccati differential equation (17) with arbitrary $b(t), T_{0}$, and $T^{*}$, where $T \leq T_{0}<T^{*}$.

Proof. Let $\varphi(t)$ and $\psi(t), \varphi, \psi \in C^{1}\left(\left(T_{0}, T^{*}\right), \mathbb{R}\right) \cap C\left(\left[T_{0}\right.\right.$, $\left.\left.T^{*}\right), \mathbb{R}\right)$, be, respectively, sub- and supersolution of (17); that is, they satisfy (18). It is not difficult to check that $h(t, u):=$ $a_{1}(t) u^{2 m}+a_{2}(t) u^{2 n}+b(t)$ is a locally Lipschitz function in the second variable. Indeed, for any bounded interval $I_{0} \subseteq$ $\left[t_{0}, \infty\right), M>0$, for all $t \in I_{0}$ and $u_{1}, u_{2} \in[-M, M]$, we have

$$
\begin{aligned}
& \left|h\left(t, u_{1}\right)-h\left(t, u_{2}\right)\right| \\
& \leq\left|a_{1}(t)\right|\left|u_{1}^{2 m}-u_{2}^{2 m}\right|+\left|a_{2}(t)\right|\left|u_{1}^{2 n}-u_{2}^{2 n}\right| \\
& \quad=\left|a_{1}(t)\right|\left|u_{1}-u_{2}\right|\left|\sum_{j=1}^{2 m} u_{1}^{2 m-j} u_{2}^{j-1}\right| \\
& \quad+\left|a_{2}(t)\right|\left|u_{1}-u_{2}\right|\left|\sum_{j=1}^{2 n} u_{1}^{2 n-j} u_{2}^{j-1}\right| \\
& \leq 2 m M^{2 m-1}\left|a_{1}(t)\right|\left|u_{1}-u_{2}\right|+2 n M^{2 n-1}\left|a_{2}(t)\right|\left|u_{1}-u_{2}\right| \\
& \leq 2 C\left(m M^{2 m-1}+n M^{2 n-1}\right)\left|u_{1}-u_{2}\right|,
\end{aligned}
$$


where $C=\max \left\{\sup _{I_{0}}\left|a_{1}(t)\right|, \sup _{I_{0}}\left|a_{2}(t)\right|\right\}$. Hence, Lemma 19 can be applied to $\varphi(t)$ and $\psi(t)$. If we set $\widetilde{\varphi}(t):=\varphi(t)$, and $\widetilde{\psi}(t):=\psi(t)$, then statement (48) is fulfilled because of assumption (18), and therefore, the desired conclusion (19) immediately follows from (49).

Corollary 22. If $a_{1}(t)$ and $a_{2}(t)$ are two continuous functions on $[T, \infty)$, then comparison principle (19) holds for the Riccati differential equation (17) with arbitrary $b(t), T_{0}$, and $T^{*}$, where $T \leq T_{0}<T^{*}$

Proof. Since $a_{1}(t)$ and $a_{2}(t)$ are two continuous functions on $[T, \infty)$, they are also locally bounded functions on $\left[t_{0}, \infty\right)$, and hence, this corollary immediately follows from Lemma 21.

Next, we present an essential lemma in which we construct a subsolution $\varphi(t)$ of (17) which has a blow-up desired property.

Lemma 23. Let $a_{1}(t) \geq 0, a_{2}(t) \geq 0$, and $b(t)$ be three arbitrary functions, and let assumption (14) hold, where $a(t)=$ $a_{1}(t)+a_{2}(t)$ if $m=n=1$ and $a(t)=\min \left\{a_{1}(t), a_{2}(t)\right\}$ otherwise. Let $\psi \in C^{1}((T, \infty), \mathbb{R}) \cap C([T, \infty), \mathbb{R})$ be a supersolution of the Riccati differential equation (17). Then, there are two real numbers $T_{0}$ and $T^{*}, T \leq T_{0}<T^{*}$, and a subsolution $\varphi \in C^{1}\left(\left(T_{0}, T^{*}\right), \mathbb{R}\right) \cap C\left(\left[T_{0}, T^{*}\right), \mathbb{R}\right)$ of $(17)$ satisfying

$$
\varphi\left(T_{0}\right) \leq \psi\left(T_{0}\right), \quad \lim _{t \rightarrow T^{*}} \varphi(t)=\infty .
$$

Proof. In particular from (14), we obtain a sequence $t_{n} \rightarrow \infty$ as $n \rightarrow \infty$ such that

$$
\int_{T_{1}}^{t_{n}} E(\tau) d \tau \longrightarrow \infty \text { as } n \longrightarrow \infty,
$$

where $T_{1}$ (determined in (14)) can be chosen so that $T_{1} \geq T$. From the previous statement, we conclude that there is a $T_{2}>$ $T_{1}$ such that

$$
\int_{T_{1}}^{T_{2}} E(\tau) d \tau=\pi
$$

Since $\int_{T_{1}}^{t} E(\tau) d \tau$ is a continuous function in the variable $t$, there is a $T_{0} \in\left[T_{1}, T_{2}\right)$ such that

$$
\int_{T_{1}}^{T_{0}} E(\tau) d \tau=0, \quad \int_{T_{1}}^{t} E(\tau) d \tau \geq 0 \quad \forall t \in\left[T_{0}, T_{2}\right) .
$$

Consequently, we derive that

$$
\begin{aligned}
\int_{T_{1}}^{t} E(\tau) d \tau & =\int_{T_{1}}^{T_{0}} E(\tau) d \tau+\int_{T_{0}}^{t} E(\tau) d \tau \\
& =\int_{T_{0}}^{t} E(\tau) d \tau, \quad t \in\left[T_{0}, T_{2}\right),
\end{aligned}
$$

which together with (53) and (54) shows

$$
\int_{T_{0}}^{t} E(\tau) d \tau \geq 0 \quad \forall t \in\left[T_{0}, T_{2}\right), \quad \int_{T_{0}}^{T_{2}} E(\tau) d \tau=\pi .
$$

Next, let $s_{0} \in(-\pi / 2, \pi / 2)$ be such that $\tan \left(s_{0}\right)=\psi\left(T_{0}\right)$, where $T_{0}$ is from (54)-(56). Such $s_{0}$ exists since the tangent function is a bijection from $(-\pi / 2, \pi / 2)$ to $\mathbb{R}$. Let

$$
V(t):=\int_{T_{0}}^{t} E(\tau) d \tau, \quad t \in\left[T_{0}, T_{2}\right] .
$$

Because of (56), we have $s_{0}+V(t)>-\pi / 2, t \in\left[T_{0}, T_{2}\right), s_{0}+$ $V\left(T_{0}\right)<\pi / 2$ and $s_{0}+V\left(T_{2}\right)>\pi / 2$. Since $V(t)$ is a continuous function, it implies the existence of a $T^{*} \in\left(T_{0}, T_{2}\right)$ such that

$$
s_{0}+V\left(T^{*}\right)=\frac{\pi}{2}, \quad-\frac{\pi}{2}<s_{0}+V(t)<\frac{\pi}{2} \quad \forall t \in\left[T_{0}, T^{*}\right) .
$$

As a consequence, the function

$$
\varphi(t)=\tan \left(s_{0}+V(t)\right), \quad t \in\left[T_{0}, T^{*}\right)
$$

is well defined and obviously satisfies

$$
\begin{aligned}
& \varphi\left(T_{0}\right)=\tan \left(s_{0}+V\left(T_{0}\right)\right)=\tan \left(s_{0}\right)=\psi\left(T_{0}\right), \\
& \lim _{t \rightarrow T^{*}} \varphi(t)=\tan \left(s_{0}+V\left(T^{*}\right)\right)=\tan \left(\frac{\pi}{2}\right)=\infty .
\end{aligned}
$$

Also, since $E(t)$ is continuous on $\left[t_{0}, \infty\right)$, we have $\varphi \in$ $C^{1}\left(\left(T_{0}, T^{*}\right), \mathbb{R}\right) \cap C\left(\left[T_{0}, T^{*}\right), \mathbb{R}\right)$. Now, by taking the derivative of $\varphi(t)$ for every $t \in\left[T_{0}, T^{*}\right)$, we obtain

$$
\begin{aligned}
\varphi^{\prime} & =\frac{1}{\cos ^{2}\left(s_{0}+\int_{T_{0}}^{t} E(\tau) d \tau\right)} \cdot E(t) \\
& =E(t)\left(1+\tan ^{2}\left(s_{0}+\int_{T_{0}}^{t} E(\tau) d \tau\right)\right) \\
& =E(t) \varphi^{2}+E(t) .
\end{aligned}
$$

According to (14), we observe that

(1) if $m=n=1$, then

$$
\begin{aligned}
E(t) \varphi^{2}+E(t) & \leq\left(a_{1}(t)+a_{2}(t)\right) \varphi^{2}+b(t) \\
& =a_{1}(t) \varphi^{2 m}+a_{2}(t) \varphi^{2 n}+b(t) ;
\end{aligned}
$$

(2) if $1=\min \{m, n\}<\max \{m, n\}$, then

$$
\begin{aligned}
E(t) \varphi^{2}+E(t) & \leq a(t) \varphi^{2}+b(t) \leq a(t)\left(\varphi^{2 m}+\varphi^{2 n}\right)+b(t) \\
& \leq a_{1}(t) \varphi^{2 m}+a_{2}(t) \varphi^{2 n}+b(t) ;
\end{aligned}
$$

(3) if $\min \{m, n\}>1$, then

$$
\begin{aligned}
E(t) \varphi^{2}+E(t) & \leq a(t) \varphi^{2}+b(t)-a(t) \\
& \leq a(t)\left(\varphi^{2 m}+\varphi^{2 n}+1\right)+b(t)-a(t) \\
& \leq a_{1}(t) \varphi^{2 m}+a_{2}(t) \varphi^{2 n}+b(t) .
\end{aligned}
$$


Thus, in all three cases of $m, n \in \mathbb{N}$, we have

$$
E(t) \varphi^{2}+E(t) \leq a_{1}(t) \varphi^{2 m}+a_{2}(t) \varphi^{2 n}+b(t), \quad t \in\left[T_{0}, T^{*}\right) .
$$

Putting the previous inequality into (61) and taking into account of (60), we conclude that

$$
\begin{gathered}
\varphi\left(T_{0}\right) \leq \psi\left(T_{0}\right), \quad \varphi^{\prime} \leq a_{1}(t) \varphi^{2 m}+a_{2}(t) \varphi^{2 n}+b(t), \\
t \in\left[T_{0}, T^{*}\right), \quad \lim _{t \rightarrow T^{*}} \varphi(t)=\infty .
\end{gathered}
$$

It proves that $\varphi(t)$ is a subsolution of the Riccati differential equation (17) which satisfies the statement (51).

Next, we are concerned with the following technical but crucial lemma.

Lemma 24. Let the assumptions of Theorem 5 in the cases (i)-(iii) hold. If the main equation (1) allows a nonoscillatory solution $x(t)$, then the function $\psi(t)$ given by (21) is welldefined with respect to such an $x(t)$ and some $T \geq t_{0}, \psi \in$ $C^{1}((T, \infty), \mathbb{R}) \cap C([T, \infty), \mathbb{R})$, and $\psi(t)$ is a supersolution of the Riccati differential equation (17).

Proof. If the main equation (1) allows a nonoscillatory solution $x(t)$, then there is a $T \geq t_{0}$ such that $x(t) \neq 0$ for all $t \geq T$. Hence, the function $\psi(t)$ given by $(21)$ is well defined for such an $x(t)$. Next, making the derivative of $\psi(t)$, using that $x(t)$ satisfies (1) and taking common assumptions of Theorem 5 for the functions $p(t), r(t), q(t), k_{1}(u, v)$, and $k_{2}(u, v)$, we obtain

$$
\begin{aligned}
\psi^{\prime}(t)= & -\frac{1}{x(t)}\left(r(t) k_{1}\left(x(t), x^{\prime}(t)\right)\right)^{\prime} \\
& +\frac{1}{x^{2}(t)} r(t) k_{1}\left(x(t), x^{\prime}(t)\right) x^{\prime}(t) \\
= & \frac{p(t)}{x(t)} k_{2}\left(x(t), x^{\prime}(t)\right) x^{\prime}(t) \\
& +\frac{r(t)}{x^{2}(t)} k_{1}\left(x(t), x^{\prime}(t)\right) x^{\prime}(t)+q(t) \frac{f(x(t))}{x(t)} \\
= & \frac{p(t)}{x^{2 n}(t)}\left[k_{2}\left(x(t), x^{\prime}(t)\right) x^{2 n-1}(t) x^{\prime}(t)\right] \\
& +\frac{r(t)}{x^{2 m}(t)}\left[k_{1}\left(x(t), x^{\prime}(t)\right) x^{2 m-2}(t) x^{\prime}(t)\right] \\
& +q(t) \frac{f(x(t))}{x(t)} .
\end{aligned}
$$

Depending on each of the three cases (i)-(iii) of Theorem 5, from the previous equality, we obtain $\psi^{\prime}(t) \geq \begin{cases}\frac{\alpha_{2} p(t)}{x^{2 n}(t)} k_{1}^{2 n}\left(x(t), x^{\prime}(t)\right) & \\ +\frac{r(t)}{\alpha_{1} x^{2 m}(t)} k_{1}^{2 m}\left(x(t), x^{\prime}(t)\right)+K q(t), & \text { in (i)-Theorem 5, } \\ \frac{\alpha_{2} p(t)}{x^{2 n}(t)} k_{1}^{2 n}\left(x(t), x^{\prime}(t)\right)+K q(t), & \text { in (ii)-Theorem 5, } \\ \frac{r(t)}{\alpha_{1} x^{2 m}(t)} k_{1}^{2 m}\left(x(t), x^{\prime}(t)\right)+K q(t), & \text { in (ii)-Theorem 5, }\end{cases}$

Next from (21), we also have

$$
k_{1}\left(x(t), x^{\prime}(t)\right)=-\frac{x(t)}{r(t)} \psi(t)
$$

Now, from (68) and (69), we immediately obtain: $\psi^{\prime} \geq$ $a_{1}(t) \psi^{2 m}+a_{2}(t) \psi^{2 n}+b(t), t \geq T$. According to the definition of a supersolution, the previous inequality shows this lemma.

Lemma 25. Let the assumptions of Theorem 5 in the cases (iv)-(v) hold. If the main equation (1) allows a nonoscillatory solution $x(t)$, then the function $\psi(t)$ given by

$$
\psi(t)= \begin{cases}-\frac{r(t) k_{1}\left(x(t), x^{\prime}(t)\right)}{x(t)} & \\ x e^{\alpha_{2} \int(p(\tau) / r(\tau)) d \tau,} \quad t \geq T, & \text { in the case (iv), } \\ -\frac{r(t) k_{1}\left(x(t), x^{\prime}(t)\right)}{x(t)} & \\ -\frac{\alpha_{1} \alpha_{2} p(t)}{2}, \quad t \geq T, & \text { in the case (v) }\end{cases}
$$

is well defined with respect to such an $x(t)$ and some $T \geq t_{0}$ such that $\psi \in C^{1}((T, \infty), \mathbb{R}) \cap C([T, \infty), \mathbb{R})$, and $\psi(t)$ is a supersolution of the Riccati differential equation (17).

The proof of Lemma 25 is omitted because it is very similar to the proof of the following lemma.

Lemma 26. Let assumptions of Theorem 15 hold. If the main equation (1) allows a nonoscillatory solution $x(t)$, then the function $\psi(t)$ given by

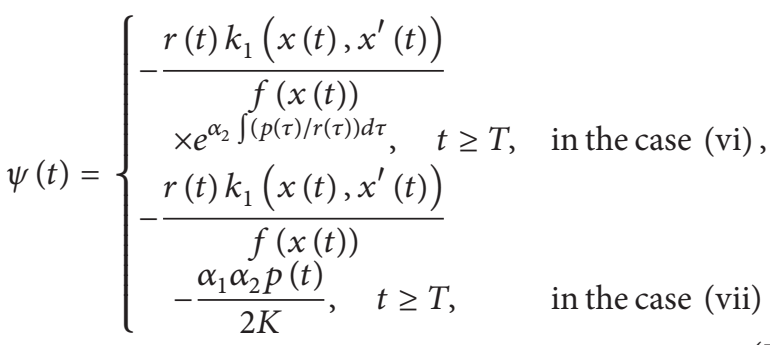

is well defined with respect to such an $x(t)$ and some $T \geq t_{0}, \psi \in$ $C^{1}((T, \infty), \mathbb{R}) \cap C([T, \infty), \mathbb{R})$, and $\psi(t)$ is a supersolution of the Riccati differential equation (17), where $a(t)=a_{1}(t)+a_{2}(t)$ and $a(t), b(t)$ are given in the case (vi) of Theorem 5 . 
Proof. Let $x(t)$ be a nonoscillatory solution of (1), and thus, we can take a $T \geq t_{0}$ such that $x(t) \neq 0$ on $[T, \infty)$. Let $\psi_{0}(t)$ be a function defined by

$$
\psi_{0}(t)=-\frac{r(t) k_{1}\left(x(t), x^{\prime}(t)\right)}{f(x(t))}, \quad t \geq T .
$$

From the assumptions of Theorem 15 and from equalities (1) and (72), we can easily make the following computation:

$$
\begin{aligned}
\psi_{0}^{\prime}(t)= & -\frac{1}{f(x(t))}\left(r(t) k_{1}\left(x(t), x^{\prime}(t)\right)\right)^{\prime} \\
& +\frac{1}{f^{2}(x(t))} r(t) k_{1}\left(x(t), x^{\prime}(t)\right) x^{\prime}(t) f^{\prime}(x(t)) \\
= & \frac{p(t)}{f(x(t))}\left[k_{2}\left(x(t), x^{\prime}(t)\right) x^{\prime}(t)\right] \\
& +\frac{r(t)}{f^{2}(x(t))}\left[k_{1}\left(x(t), x^{\prime}(t)\right) x^{\prime}(t)\right] f^{\prime}(x(t))+q(t) \\
\geq & \alpha_{2} p(t)\left[\frac{k_{1}\left(x(t), x^{\prime}(t)\right)}{f(x(t))}\right] \\
& +\frac{K r(t)}{\alpha_{1}}\left[\frac{k_{1}\left(x(t), x^{\prime}(t)\right)}{f(x(t))}\right]^{2}+q(t) \\
= & -\frac{\alpha_{2} p(t)}{r(t)} \psi_{0}(t)+\frac{K}{\alpha_{1} r(t)} \psi_{0}^{2}(t)+q(t)
\end{aligned}
$$

that is,

$$
\psi_{0}^{\prime}(t) \geq \frac{K}{\alpha_{1} r(t)} \psi_{0}^{2}(t)-\frac{\alpha_{2} p(t)}{r(t)} \psi_{0}(t)+q(t), \quad t>T .
$$

Now, if the middle term on the right-hand side of (74) is moved into the left-hand side, and multiplying such equality by $e^{\alpha_{2} \int(p(\tau) / r(\tau)) d \tau}$, we conclude that the function

$$
\psi(t)=\psi_{0}(t) e^{\alpha_{2} \int p(\tau) / r(\tau) d \tau}, \quad t \geq T
$$

satisfies the Riccati differential equation (17) with respect to $a(t)$ and $b(t)$ given in the case (vi) of Theorem 15, which proves the first statement of this lemma.

However, if we group the first two terms on the right-hand side of (74) by the purpose of getting the complete square, then from (74) we easily conclude that the function:

$$
\psi(t)=\psi_{0}(t)-\frac{\alpha_{1} \alpha_{2} p(t)}{2 K}, \quad t \geq T
$$

satisfies the Riccati differential equation (17) with respect to $a(t)$ and $b(t)$ given in the case (vii) of Theorem 15, which proves the second statement of this lemma.

Now, we are able to present a common proof of the main results of the paper.
Proof of Theorems 5 and 15. At first, it is worth pointing out that the functions: $a(t), a_{1}(t), a_{2}(t)$, and $b(t)$, which are appearing at the same time in the main assumption (14) and the Riccati differential equation (17), only depend on the appropriate combination of basic assumptions on the coefficients: $r(t) p(t)$, and $q(t)$ and the functions: $k_{1}(u, v)$ and $k_{2}(u, v)$, which are formulated in one of the five cases of Theorem 5 and one of the two cases of Theorem 15.

Now, if we assume the contrary to the main assertion of the theorem; that is, if (1) is not oscillatory, then there is a nonoscillatory solution $x(t)$ of (1) and a point $T \geq t_{0}$ and $T \geq T_{1}$, where $T_{1}$ is appearing in (14), such that $x(t) \neq 0$ for all $t \in[T, \infty)$. Then by Lemmas 24, 25 and 26, the function $\psi(t)$ given by (21) or (70), and (71) is well defined with respect to such an $x(t)$, smooth enough on $(T, \infty)$, and it is a supersolution of the Riccati differential equation (17). Taking into account the main results of Lemma 23, we obtain the two numbers $T_{0}$ and $T^{*}, T \leq T_{0}<T^{*}$, and a subsolution $\varphi(t)$ of (17) such that the blow-up argument (51) is satisfied. By Corollary 22, we can apply the comparison principle (19) to (17) with arbitrary $T_{0}$ and $T^{*}$, where $T \leq T_{0}<T^{*}$. Hence, combining (19) and (51), we get $\psi(t) \rightarrow \infty$ as $t \rightarrow T^{*}$, which contradicts the fact that $\psi \in C^{1}\left(\left(T_{0}, \infty\right), \mathbb{R}\right) \cap C\left(\left[T_{0}, \infty\right), \mathbb{R}\right)$. Thus, $\psi(t)$ is not possible, and therefore, (1) does not allow any nonoscillatory solution.

Proof of Lemma 19. Let $\widetilde{d}(t)=\widetilde{\psi}(t)-\widetilde{\varphi}(t)$ and $\widetilde{\varphi}\left(T_{0}\right) \leq \widetilde{\psi}\left(T_{0}\right)$; that is,

$$
\widetilde{d}\left(T_{0}\right) \geq 0 .
$$

If statement (49) does not hold, then there is a point $T_{*} \in$ $\left(T_{0}, T^{*}\right)$ such that $\tilde{\varphi}\left(T_{*}\right)>\widetilde{\psi}\left(T_{*}\right)$; that is,

$$
\tilde{d}\left(T_{*}\right)<0 .
$$

Moreover, since $\tilde{d} \in C^{1}\left(\left(T_{0}, T^{*}\right), \mathbb{R}\right) \cap C\left(\left[T_{0}, T^{*}\right), \mathbb{R}\right)$ from (77) and (78), we obtain a $T_{1} \in\left[T_{0}, T_{*}\right)$ such that

$$
\widetilde{d}\left(T_{1}\right)=0, \quad \widetilde{d}(t)<0 \quad \forall t \in\left(T_{1}, T_{*}\right] .
$$

Since $\tilde{\varphi}, \tilde{\psi} \in C\left(\left[T_{1}, T_{*}\right]\right)$, we may use (47) in particular for

$$
I_{0}=\left[T_{1}, T_{*}\right], \quad M=\max \left\{\max _{t \in I_{0}}|\widetilde{\varphi}(t)|, \max _{t \in I_{0}}|\tilde{\psi}(t)|\right\} .
$$

Hence, from (47), (48), and (79), we get

$$
\begin{aligned}
\tilde{d}^{\prime}(t)=\widetilde{\psi}^{\prime}(t)-\widetilde{\varphi}^{\prime}(t) & \geq h(t, \widetilde{\psi}(t))-h(t, \widetilde{\varphi}(t)) \\
& \geq-L|\widetilde{d}(t)|=L \widetilde{d}(t), \quad t \in\left(T_{1}, T_{*}\right) .
\end{aligned}
$$

Multiplying this inequality by $e^{-L t}$ and denoting by $\theta(t):=$ $\widetilde{d}(t) e^{-L t}$, we get

$$
\theta^{\prime}(t)=e^{-L t}(\widetilde{d}(t)-L \widetilde{d}(t)) \geq 0, \quad t \in\left(T_{1}, T_{*}\right) .
$$

Thus, according to (79) and (82), we have that $\theta\left(T_{1}\right)=0$, $\theta(t)<0$ and $\theta^{\prime}(t) \geq 0$ on $\left(T_{1}, T_{*}\right)$, which is not possible. Hence, the hypothesis (78) yields to a contradiction and, thus, $\widetilde{\varphi}(t) \leq \widetilde{\psi}(t)$ for all $t \in\left[T_{0}, T^{*}\right)$. 


\section{Acknowledgment}

This work is supported by the Scientific Project of the Ministry of Science and Education of Croatia no. 036-03616211291.

\section{References}

[1] A. Zhao, Y. Wang, and J. Yan, "Oscillation criteria for secondorder nonlinear differential equations with nonlinear damping," Computers \& Mathematics with Applications, vol. 56, no. 2, pp. 542-555, 2008.

[2] S. P. Rogovchenko and Y. V. Rogovchenko, "Oscillation theorems for differential equations with a nonlinear damping term," Journal of Mathematical Analysis and Applications, vol. 279, no. 1, pp. 121-134, 2003.

[3] A. Tiryaki and A. Zafer, "Oscillation of second-order nonlinear differential equations with nonlinear damping," Mathematical and Computer Modelling, vol. 39, no. 2-3, pp. 197-208, 2004.

[4] R. P. Agarwal and Q. R. Wang, "Oscillation and asymptotic behavior for second-order nonlinear perturbed differential equations," Mathematical and Computer Modelling, vol. 39, no. 13, pp. 1477-1490, 2004.

[5] J. W. Baker, "Oscillation theorems for a second order damped nonlinear differential equation," SIAM Journal on Applied Mathematics, vol. 25, no. 1, pp. 37-40, 1973.

[6] L. E. Bobisud, "Oscillation of solutions of damped nonlinear equations," SIAM Journal on Applied Mathematics, vol. 19, no. 3, pp. 601-606, 1970.

[7] S. R. Grace, "Oscillation theorems for nonlinear differential equations of second order," Journal of Mathematical Analysis and Applications, vol. 171, no. 1, pp. 220-241, 1992.

[8] Y. Huang and F. Meng, "Oscillation criteria for forced secondorder nonlinear differential equations with damping," Journal of Computational and Applied Mathematics, vol. 224, no. 1, pp. 339-345, 2009.

[9] Q. Long and Q. R. Wang, "New oscillation criteria of secondorder nonlinear differential equations," Applied Mathematics and Computation, vol. 212, no. 2, pp. 357-365, 2009.

[10] Q. R. Wang, X. M. Wu, and S. M. Zhu, "Oscillation criteria for second-order nonlinear damped differential equations," Computers \& Mathematics with Applications, vol. 46, no. 8-9, pp. 1253-1262, 2003.

[11] N. Yamaoka, "Oscillation criteria for second-order damped nonlinear differential equations with $p$-Laplacian," Journal of Mathematical Analysis and Applications, vol. 325, no. 2, pp. 932948, 2007.

[12] X. Zhao and F. Meng, "Oscillation of second-order nonlinear ODE with damping," Applied Mathematics and Computation, vol. 182, no. 2, pp. 1861-1871, 2006.

[13] N. Shang and H. Qin, "Comments on the paper: "Oscillation of second-order nonlinear ODE with damping" [Applied Mathematics and Computation 199 (2008) 644-652]," Applied Mathematics and Computation, vol. 218, no. 6, pp. 2979-2980, 2011.

[14] Ch. G. Philos, "Oscillation theorems for linear differential equations of second order," Archiv der Mathematik, vol. 53, no. 5, pp. 482-492, 1989.

[15] J. S. W. Wong, "On Kamenev-type oscillation theorems for second-order differential equations with damping," Journal of Mathematical Analysis and Applications, vol. 258, no. 1, pp. 244257, 2001.
[16] Z. Xu and Y. Xia, "Kamenev-type oscillation criteria for secondorder quasilinear differential equations," Electronic Journal of Differential Equations, vol. 2005, pp. 1-9, 2005.

[17] J. R. Yan, "Oscillation theorems for second order linear differential equations with damping," Proceedings of the American Mathematical Society, vol. 98, no. 2, pp. 276-282, 1986.

[18] C. C. Yeh, "Oscillation theorems for nonlinear second order differential equations with damped term," Proceedings of the American Mathematical Society, vol. 84, no. 3, pp. 397-402, 1982.

[19] M. Pašić, "Isoperimetric inequalities in quasilinear elliptic equations of Leray-Lions type," Journal de Mathématiques Pures et Appliquées, vol. 75, no. 4, pp. 343-366, 1996.

[20] Y. Tian and F. Li, "Comparison results for nonlinear degenerate Dirichlet and Neumann problems with general growth in the gradient," Journal of Mathematical Analysis and Applications, vol. 378, no. 2, pp. 749-763, 2011.

[21] W. Walter, "A note on Sturm-type comparison theorems for nonlinear operators," Journal of Differential Equations, vol. 135, no. 2, pp. 358-365, 1997.

[22] W. Walter, Ordinary Differential Equations, vol. 182 of Graduate Texts in Mathematics. Readings in Mathematics, Springer, New York, NY, USA, 1998.

[23] S. Carl, "Existence and comparison results for noncoercive and nonmonotone multivalued elliptic problems," Nonlinear Analysis. Theory, Methods \& Applications A, vol. 65, no. 8, pp. 1532-1546, 2006.

[24] W. B. Fite, "Concerning the zeros of the solutions of certain differential equations," Transactions of the American Mathematical Society, vol. 19, no. 4, pp. 341-352, 1918.

[25] A. Wintner, "A criterion of oscillatory stability," Quarterly of Applied Mathematics, vol. 7, pp. 115-117, 1949.

[26] W. Leighton, "The detection of the oscillation of solutions of a second order linear differential equation," Duke Mathematical Journal, vol. 17, pp. 57-61, 1950.

[27] J. S. W. Wong, "Oscillation and nonoscillation of solutions of second order linear differential equations with integrable coefficients," Transactions of the American Mathematical Society, vol. 144, pp. 197-215, 1969.

[28] B. Travis, "Nth order extension of the Wintner-Leighton theorem," Applied Mathematics and Computation, vol. 110, no. 2-3, pp. 115-119, 2000. 


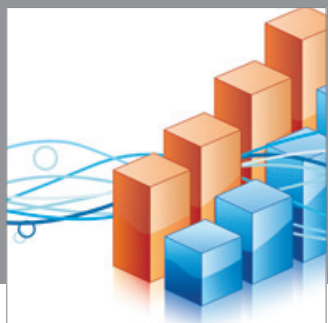

Advances in

Operations Research

mansans

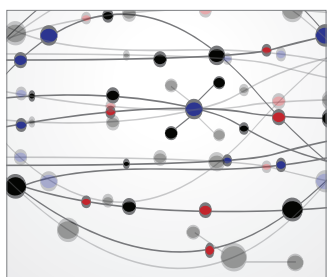

The Scientific World Journal
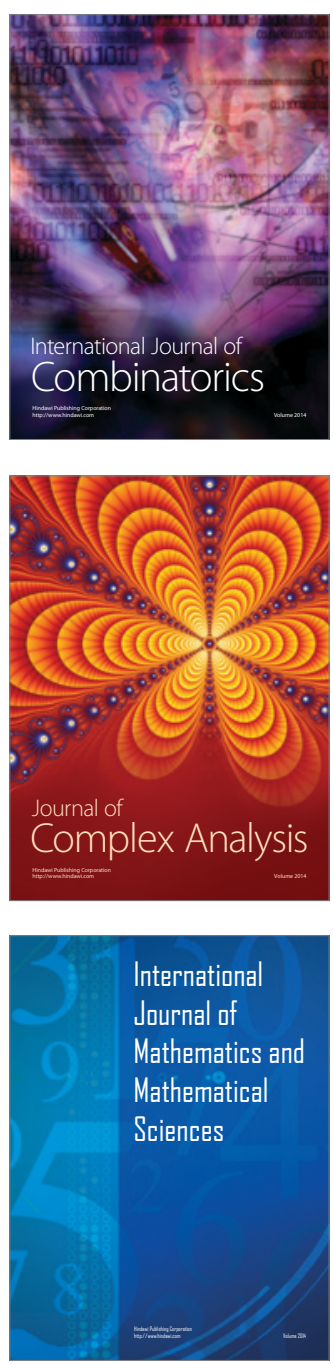
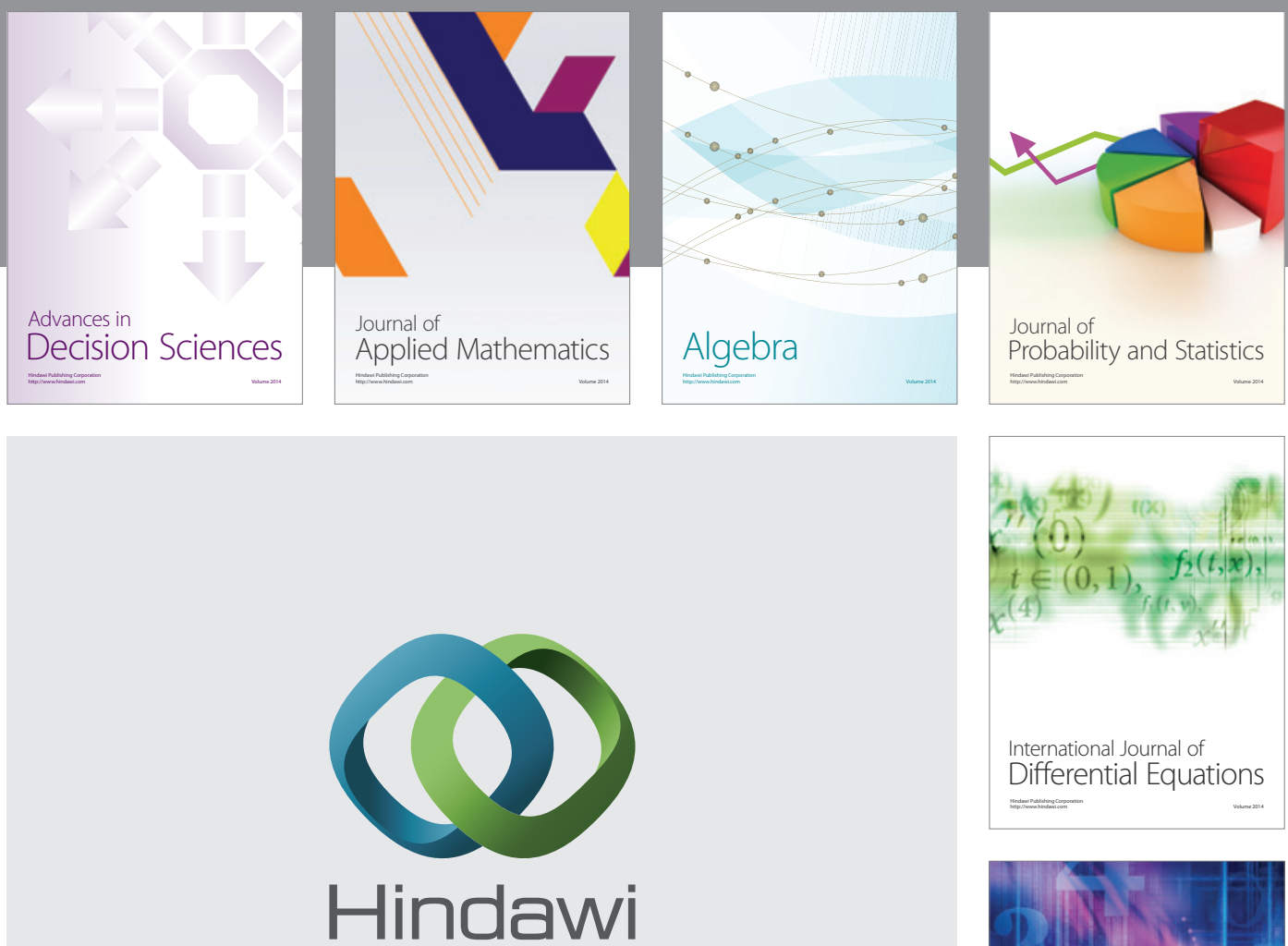

Submit your manuscripts at http://www.hindawi.com
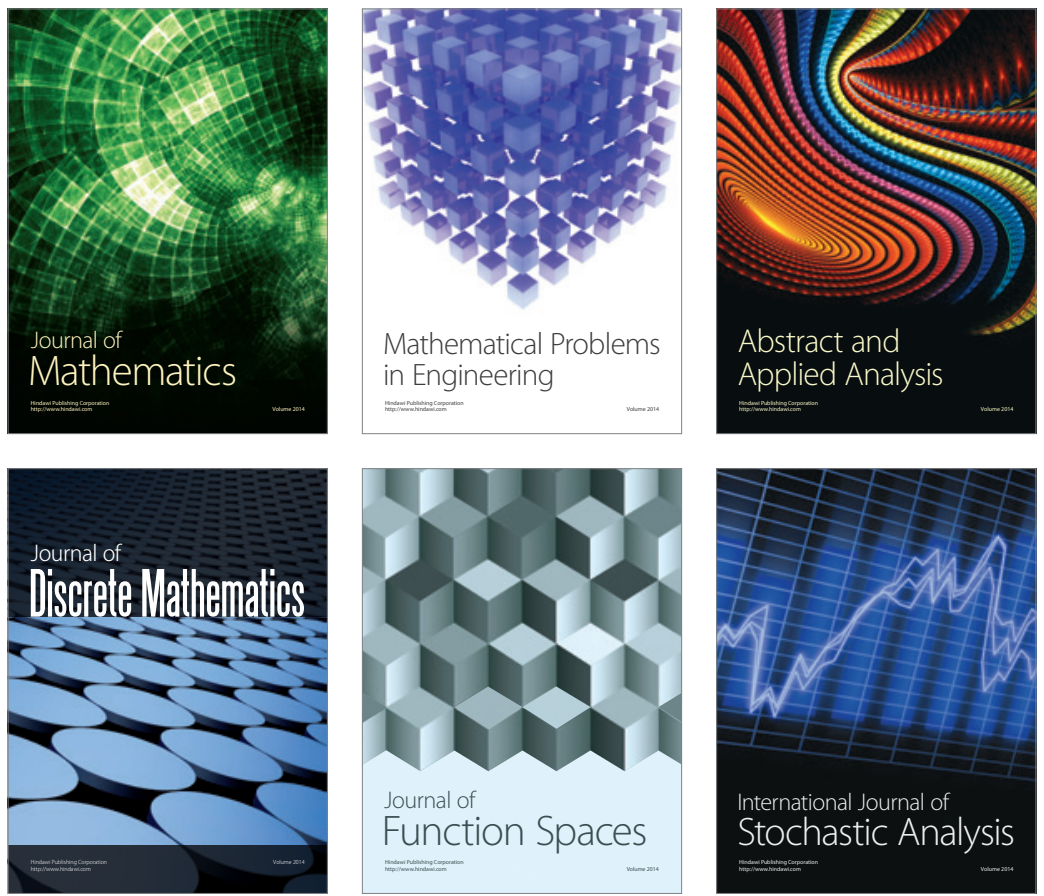

Journal of

Function Spaces

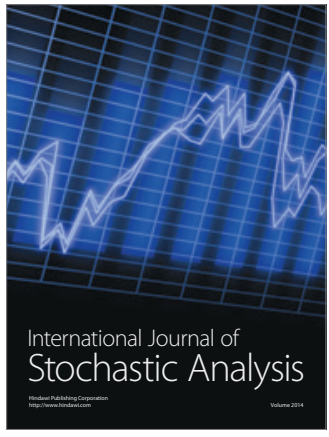

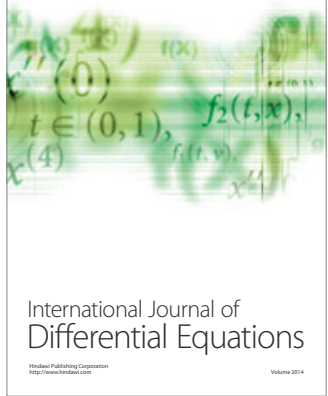
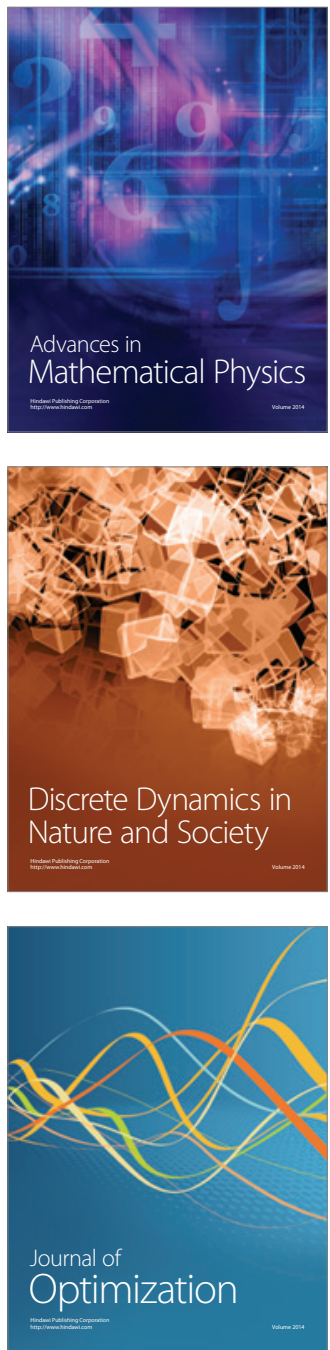